\title{
Do emotional faces capture attention, and does this depend on awareness? Evidence from the visual probe paradigm
}

Article

Accepted Version

Hedger, N., Garner, M. and Adams, W. J. (2019) Do emotional faces capture attention, and does this depend on awareness? Evidence from the visual probe paradigm. Journal of Experimental Psychology: Learning, Memory \& Cognition, 45 (6). pp. 790-802. ISSN 0278-7393 doi:

https://doi.org/10.1037/xhp0000640 Available at https://centaur.reading.ac.uk/87285/

It is advisable to refer to the publisher's version if you intend to cite from the work. See Guidance on citing.

To link to this article DOI: http://dx.doi.org/10.1037/xhp0000640

Publisher: American Psychological Association

All outputs in CentAUR are protected by Intellectual Property Rights law, including copyright law. Copyright and IPR is retained by the creators or other copyright holders. Terms and conditions for use of this material are defined in the End User Agreement. 


\section{CentAUR}

Central Archive at the University of Reading

Reading's research outputs online 
See discussions, stats, and author profiles for this publication at: https://www.researchgate.net/publication/330934689

\section{Do Emotional Faces Capture Attention, and Does This Depend on Awareness? Evidence From the Visual Probe Paradigm}

Article in Journal of Experimental Psychology Human Perception \& Performance · January 2019

DOI: $10.1037 /$ hhp 0000640

\section{CITATIONS}

3 authors:

Nicholas Hedger

University of Reading

19 PUBLICATIONS 232 CITATIONS

SEE PROFILE

Wendy Jo Adams

University of Southampton

99 PUBLICATIONS 1,198 CITATIONS

SEE PROFILE

Some of the authors of this publication are also working on these related projects:

Project Perceptual Aftereffects and Attention View project

Project Visual Illusions and Awareness View project
190

University of Southampton

104 PUBLICATIONS 2,207 CITATIONS

SEE PROFILE 
EMOTION AND ATTENTION

1

2

3

4

5

6 Do Emotional Faces Capture Attention, and Does this Depend on Awareness? Evidence from 7 the Visual Probe Paradigm.

8

9

Nicholas Hedger ${ }^{\mathrm{a}}$, Matthew Garner ${ }^{\mathrm{b}, \mathrm{c}}$, Wendy J. Adams ${ }^{\mathrm{b}}$

10

11

$12{ }^{a}$ Psychology, University of Reading, Reading, RG6 6AL, UK

$13{ }^{\mathrm{b}}$ Centre for Vision and Cognition, Psychology, University of Southampton,

14 Southampton, SO17 1BJ, UK

$15{ }^{\mathrm{c}}$ Clinical and Experimental Sciences, Faculty of Medicine, University of Southampton,

16 Southampton, SO17 1BJ, UK

17

18

19

20 Corresponding author: Nicholas Hedger.

21 Email: N.Hedger@reading.ac.uk

22 Phone: +447742142858

23 Address: Psychology, Reading, Reading, RG6 6AL, UK.

24

25 Word Count: 8294 
EMOTION AND ATTENTION

26

27

28 
EMOTION AND ATTENTION

29

30

31 The visual probe (VP) paradigm provides evidence that emotional stimuli attract attention.

32 Such effects have been reported even when stimuli are presented outside of awareness. These

33 findings have shaped the idea that humans possess a processing pathway that detects

34 evolutionarily significant signals independently of awareness. Here, we addressed two unresolved questions: First, if emotional stimuli attract attention, is this driven by their affective content, or by low-level image properties (e.g. luminance contrast)? Second, does attentional capture occur under conditions of genuine unawareness? We found that observers preferentially allocated attention to emotional faces under aware viewing conditions.

However, this effect was best explained by low-level stimulus properties, rather than emotional content. When stimuli were presented outside of awareness (via continuous flash suppression or masking), we found no evidence that attention was directed towards emotional face stimuli. Finally, observer's awareness of the stimuli (assessed by d prime) predicted attentional cuing. Our data challenge existing literature: First, we cast doubt on the notion of preferential attention to emotional stimuli in the absence of awareness. Second, we question whether effects revealed by the VP paradigm genuinely reflect emotion-sensitive processes, instead suggesting they can be more parsimoniously explained by low-level variability between stimuli.

Keywords: threat; emotion; attention; awareness; visual probe 
EMOTION AND ATTENTION

50 Public Significance Statement

51 Emotionally salient stimuli (such as fearful faces) are prioritised in attention, even when they

52 are presented outside of awareness. Moreover, such effects are often found to be larger in

53 anxious populations, suggesting that emotion sensitive mechanisms that operate without

54 awareness may be involved in the aetiology/ maintenance of anxiety disorders. However, the

55 mechanisms underlying such 'emotional attention' effects remain unclear. Here we show that

56 i) emotional stimuli only attract attention under conditions where observers are aware of

57 stimuli. ii) preferential attention to emotional faces is best explained by low-level stimulus

58 properties (e.g. luminance contrast) rather than emotion-sensitive processes. Our study

59 highlights the need for careful experimental control in basic and clinical research

60 investigating the link between emotion and attention. 
EMOTION AND ATTENTION

61

62

63

64

65

66

67

68

69

70

Human visual perception has limited capacity and must direct resources towards salient stimuli, events and spatial locations. Many behavioural studies suggest that emotionally salient (particularly threatening) stimuli attract our attention (Armony \& Dolan, 2002; Ohman, Flykt \& Esteves, 2001; Vuilleumier \& Schwartz, 2001). The visual probe paradigm provides evidence of this effect. On a typical trial, an emotionally salient and a neutral target stimulus are presented on either side of a central fixation cross, before a probe (usually a small dot or arrow) appears at the location preceded by either the emotional stimulus (valid trial) or neutral stimulus (invalid trial). Observers then make a speeded response to indicate the location or orientation of the probe (left vs. right, or pointing up or down). Responses are typically faster in valid trials than invalid trials, suggesting that spatial attention has been preferentially allocated to the location of the emotional stimulus (BarHaim, Lavy, Pergamin, Bakermans-Kranenburg, \& van Ijzendoorn, 2007). There is tremendous interest in understanding the mechanisms of this selection process - how does the visual system prioritize stimuli that are most important to its survival?

Evolutionary theories suggest that humans possess an independent, sub-cortical visual pathway that operates without awareness and rapidly directs processing resources towards threatening stimuli (Garrido, 2012; Tamietto \& de Gelder, 2010). This theory has intuitive appeal - it may take hundreds of milliseconds for retinal stimulation to generate a conscious percept (Koch, 2004; Sekar, Findley, Poeppel, \& Llinás, 2013). If threats could modulate an observer's behaviour rapidly and independently of their conscious registration, survival odds would be increased (Morris, Öhman, \& Dolan, 1999). This notion is intriguing, because it suggests that there are specialised ways of (and independent neural substrates for) prioritising affective stimuli. Moreover, such an idea has influenced thinking about clinical disorders. For instance, dysfunction in the systems involved with preconscious threat detection are thought 
EMOTION AND ATTENTION

to underlie the hypersensitivity to threat and maladaptive perceptual biases exhibited by individuals with anxiety disorders (Mogg \& Bradley, 1998; Ohman \& Mineka, 2001).

Evidence for the unconscious prioritisation of threat has typically relied on measuring responses to stimuli that are presented to observers outside of awareness (Kim \& Blake, 2005). A long history of observations from paradigms such as backward masking, binocular rivalry and continuous flash suppression (CFS) has revealed that threat stimuli suppressed from awareness can nonetheless elicit adaptive changes in neural activity (Jiang \& He, 2006; Whalen et al., 2004; Williams, Morris, McGlone, Abbott, \& Mattingley, 2004) and physiological arousal (Lapate, Rokers, Li, \& Davidson, 2013; Ohman \& Soares, 1994). Behaviourally, the masked visual probe (MVP) paradigm has provided evidence that threat stimuli receive prioritized processing in the absence of awareness. In a modification of the standard visual probe design, target stimuli are presented briefly, and then replaced with a masking pattern. The small stimulus onset asynchrony (SOA) between the target and mask (usually $\sim 17$ or $\sim 33$ milliseconds) means that observers typically report perceiving the mask, but not the preceding target stimulus (Wiens \& Ohman, 2007): visual presentation of the target stimulus is dissociated from awareness of it. Thus, the MVP paradigm can be employed as a tool to examine attentional orienting to emotionally salient stimuli in the absence of their conscious registration.

In a recent meta-analysis of the MVP paradigm (Hedger, Gray, Garner, \& Adams, 2016) we found that the magnitude of threat-related bias (i.e. the valid vs. invalid response time (RT) difference) across all stimulus types (including fear and angry faces, negative words and images from the International Affective Picture System) tends to be small (Cohen's $d_{z}=0.28$ ). Our analyses also suggest that effect sizes are strongly modulated by stimulus visibility: the threat-related bias was significantly larger if the SOA between stimuli and masks was $>30 \mathrm{~ms}$ than if it was $<30 \mathrm{~ms}$. Critically, this suggests that unintended 
EMOTION AND ATTENTION

111 stimulus visibility may increase threat-related biases: many observers achieve above-chance

112 detection of $33 \mathrm{~ms}$ targets, as revealed by stringent signal detection measures of awareness

113 (Pessoa, Japee, Sturman, \& Ungerleider, 2006; Szczepanowski \& Pessoa, 2007). In addition,

114 we found that this effect of SOA on threat bias was greater within studies that did not

115 implement an awareness check to verify that masking successfully eliminated stimulus

116 visibility. Interestingly, this suggests threat related biases in the MVP paradigm could be

117 modulated by, or perhaps even driven by residual awareness of the masked stimuli.

118 In MVP studies that have measured observers' awareness of masked stimuli, this is

119 usually implemented via an independent block of trials wherein observers complete an

120 alternative forced choice (AFC) task, such as discriminating between different masked

121 stimuli (Carlson, Reinke, \& Habib, 2009; Fox, 2002; Mogg, Bradley, \& Hallowell, 1994). In

122 general, if observers' performance does not significantly exceed chance performance in this

123 control task, it is concluded that any threat biases obtained during the experimental trials can

124 be attributed to processes that occur independently of awareness of the threat stimuli.

125 Establishing null sensitivity to stimuli via a forced choice task in this way is

126 associated with formidable practical and conceptual issues (Wiens, 2008). For instance,

127 awareness checks in the MVP paradigm have typically lacked statistical power, i.e. the

128 likelihood of type II errors (failure to detect an observer's residual discrimination of target

129 stimuli) may have been problematically high. Our meta analysis revealed that, on average,

130 across MVP experiments, observers were classed as unaware of stimuli if 2AFC performance

131 was less than $68 \%$. Importantly, this permits deviations from chance performance that are

132 moderate in magnitude (Cohen's $h=0.38$, see Cohen, 1977), which invalidates strong

133 statements about truly 'unconscious' processing of the masked stimuli. Another statistical

134 issue is that if observers are selected post-hoc on the basis of chance-level performance in an

135 awareness check, then this can bias evidence in favour of unconscious processing - reflecting 
EMOTION AND ATTENTION

136 a statistical principle referred to as 'regression to the mean' (Shanks, 2016). Therefore, it is

137 important to assess not only this subset of observers, but also to consider whether individual-

138 level awareness of stimuli predicts attentional bias across the full sample of participants.

139 Moreover, it is important to note that only one study employed a signal detection measure

140 (d'- d prime) that corrected for individual response bias (Koster, Verschuere, Burssens,

141 Custers, \& Crombez, 2007). Taken together, these limitations suggest that more rigorous

142 methods are needed to assess awareness ${ }^{1}$.

143 Another interesting question, receiving increased attention, is whether any

144 behavioural effects of 'unconsciously' presented stimuli depend on the method used to

145 manipulate awareness. For instance, it is possible that threat stimuli can modulate attention

146 independently of awareness, but that the brevity of masked presentations degrades processing

147 of the target stimuli such that any attentional modulation is reduced and hard to detect.

148 Masking necessitates presentation times that are substantially briefer $(<40 \mathrm{~ms})$ than those

149 chosen to optimise attentional cueing effects in standard, supraliminal versions of the visual

150 probe task (usually around $500 \mathrm{~ms}$; Bar-Haim, et al., 2007). Since the presentation of stimuli

151 in the masked version of the visual probe paradigm is an order of magnitude briefer than in

152 the standard version, this confounds any comparison between aware and unaware processing.

153 A more direct comparison would require that subliminal stimuli are not so temporally

154 disadvantaged, relative to a supraliminal counterpart. Continuous flash suppression (CFS),

155 which is an increasingly popular method in the study of unconscious processing (Sklar et al.,

156 2012) may provide one solution to this problem. In CFS, stimuli presented to one eye can be

157 suppressed from awareness by presenting a dynamic noise pattern to the other eye (Tsuchiya

$158 \&$ Koch, 2005). With appropriate presentation parameters, CFS can render stimuli invisible

159 for several seconds, allowing time for unconscious processes to engage with the suppressed 
EMOTION AND ATTENTION

160 stimuli. The use of CFS in a visual probe paradigm may therefore provide a more suitable

161

162

163

164

165

166

167

168

169

170

171

172

173

174

175

176

177

178

179

180

181

182

183

comparison between unaware and aware states.

Finally, a critical conceptual issue concerns the stimulus attributes that drive the prioritisation of threat stimuli across all paradigms: standard visual probe, masked visual probe and CFS. Although it has been demonstrated that certain classes of threat stimuli, such as fearful faces, are reliably prioritized, one idea gaining traction is that this prioritization may be better explained by their low-level properties than by threat-sensitive processes (Gray, Adams, Hedger, Newton, \& Garner, 2013; Hedger, Adams, \& Garner, 2015b; Stein \& Sterzer, 2012). For instance, Lee and colleagues (2013) found that the increased luminance contrast resulting from the greater exposure of the scleral field (eye whites) in fearful faces (relative to neutral faces) was a good predictor of enhanced performance in an attentional cuing task. More recently, work from our own lab revealed that the relationship between a face's amplitude spectrum and the human contrast sensitivity function was a better predictor of the face's detectability in masking and CFS tasks than its perceived valence or arousal (Hedger et al., 2015b). For example, fear faces have greater luminance contrast at the spatial scales humans are sensitive to than angry faces, and this predicts their higher levels of detection. This sensory advantage of the fear expression is particularly important, since fearful faces give rise to the largest, most reliable threat-related biases in the MVP paradigm of all stimulus types (Hedger et al., 2016). As highlighted in our meta analyses, it is critical that researchers provide adequate stimulus controls such that threat-related biases driven by the semantic content of stimuli (i.e. their affective content) are distinguished from effects driven by simple low-level differences between stimuli. If processing advantages are driven by low-level stimulus properties, this negates the need to invoke unconscious processes sensitive to threat. 
EMOTION AND ATTENTION

184

185

186

187

188

189

190

191

192

193

194

195

196

197

198

199

200

201

202

203

204

205

206

207

208

The current study aims to address contentious or unresolved issues within the current literature. Specifically, we ask: (i) Do emotionally salient stimuli modulate attention in standard viewing conditions (i.e. with awareness of the stimuli)? (ii) Do emotional stimuli modulate attention under conditions of unawareness, as defined by stringent signal detection criteria? (iii) Are these effects modulated by the method used to render stimuli perceptually invisible? iv) Are attentional biases better explained by affective, or low-level variability across stimuli?

\section{Method}

\section{Participants}

Before recruiting participants, ethical approval for the study was obtained via the University of Southampton Research Ethics Committee (Submission ID: 17166). From our previous meta analyses (Hedger et al., 2016), we determined that 41 participants would be required to attain $95 \%$ power to detect the attentional effects observed when fear and neutral faces compete in the MVP paradigm $\left(d_{\mathrm{z}}=0.58\right)$. For this reason, data collection was terminated when 41 undergraduate students ( 9 male, $M$ age $=20.2$ years) had completed the experiment. All observers had normal or corrected-to-normal vision.

\section{Stimuli}

Stimuli were four male facial models, taken from the NimStim face set (Tottenham et al., 2009), depicting neutral, fearful and happy expressions. All stimuli were placed within an opaque elliptical mask to eliminate external features and were equated in luminance and root mean squared (RMS) contrast. Face stimuli were presented in two configurations. Normal faces were presented upright with veridical contrast polarity. Upside-down negative faces were rotated 180 degrees with reversed contrast polarity, producing an image similar to a photographic negative (see Figure 1). These manipulations severely disrupt the recognition 
EMOTION AND ATTENTION

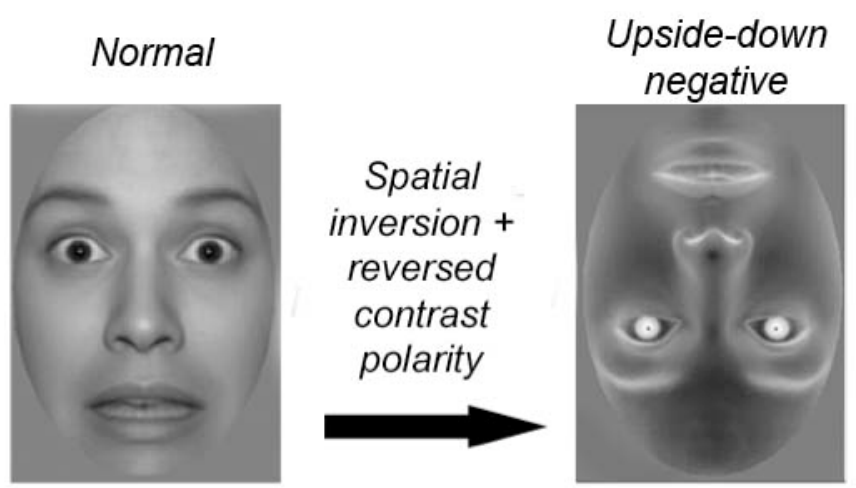

209 Figure 1. Example face stimulus presented in the normal and upside-down negative

210 configuration.

212 and affective evaluation of facial expressions (Gray et al., 2013; Hedger et al., 2015b).

213 Critically, however, these manipulations do not affect the low-level stimulus properties of the

214 image: i.e., its RMS contrast, mean luminance and amplitude spectra (and therefore the

215 energy / strength of image contours). Thus, if the valence of face images is critical in

216 directing spatial attention, we would expect any effect of expression to be reduced or

217 eliminated for the upside-down negative images, relative to the normal images (i.e. an

218 interaction between expression and stimulus configuration). Conversely, if low-level

219 properties of the stimuli explain the effect of expression, we would anticipate a similar main

220 effect of expression for both normal and upside-down negative stimuli (i.e. no interaction

221 between expression and stimulus configuration). All stimuli subtended $6.2 \times 4.1$ degrees of

222 visual angle (DVA) at the viewing distance of $70 \mathrm{~cm}$ on a $1280 \times 1024$ pixel resolution,

223 gamma corrected monitor. In all trials, observers viewed the display via a mirror stereoscope,

224 and each eye's image was framed by a random dot surround (9.5 x 11.4 DVA) to control

225 vergence.

226

227 Questionnaire Measures 
EMOTION AND ATTENTION

228

229

230

231

232

233

234

235

236

237

238

239

240

241

242

243

244

245

246

247

248

249

250

251

252

Previous work suggests that attentional biases towards emotional stimuli are

modulated by anxiety and related trait characteristics (Fox, 2002; Mogg \& Bradley, 1999).

Before the visual probe experiment, all observers completed the following measures of general and social anxiety: Trait Anxiety Inventory (STAI-T, Speilberger et al., 1983), Social Interaction Anxiety Scale (SIAS, Heimberg, Mueller, Holt, Hope, \& Liebowitz, 1992) and Social Phobia Scale (SPS, Heimberg et al., 1992).

\section{Procedure}

Each trial began with the presentation of a central fixation cross whose duration was randomly sampled from the range 300 to $1000 \mathrm{~ms}$ to avoid anticipatory responses. Observers completed 560 trials in total. On 'signal' trials (336 trials), pairs of face stimuli were presented to observers. On 'noise' trials (224 trials), no face stimuli were presented to observers; intermingling signal and noise trials enabled concurrent evaluation of stimulus awareness (see 'noise trials' section). There were three presentation conditions (Figure 2).

\section{Presentation Conditions}

\section{Standard presentation.}

In the standard presentation condition (Figure 2a - 112 trials), two faces were presented monocularly (eye of presentation counterbalanced across trials) on either side of the fixation cross for $500 \mathrm{~ms}$, whilst only the fixation cross and surround were presented to the other eye. Monocular presentation of face stimuli allowed a straightforward comparison with the CFS presentation condition. Immediately after the face presentation, a dot appeared at the location preceded by the left or right face and observers were required to report its location as quickly and accurately as possible (via left and right key button press).

\section{Masked presentation.}


EMOTION AND ATTENTION

In the masked presentation condition (Figure $2 b-112$ trials), our trial sequence

254

255

256

257

258

259

260

261

262

263

264

265

266

267

268

269

mirrored that of previous literature (Fox, 2002). Two face stimuli appeared binocularly either side of fixation for $17 \mathrm{~ms}$ before being immediately replaced by two masks (patterns of high contrast ellipses) for $17 \mathrm{~ms}$. A $17 \mathrm{~ms}$ SOA between face and mask has been commonly employed in previous MVP studies (Beaver, Mogg, \& Bradley, 2005; Fox, 2002; Koster, Verschuere, Burssens, Custers, \& Crombez, 2007; Mogg \& Bradley, 1999, 2002), due to the refresh rate of standard cathode ray tube (CRT) monitors. Immediately after presentation of the mask, a dot appeared at the location preceded by the left or right face and observers were required to report its location as quickly and accurately as possible.

\section{CFS presentation.}

In the CFS presentation condition (Figure 2c - 112 trials), two faces were presented monocularly (counterbalanced across eyes) on either side of the fixation cross for 500ms, whilst dynamic masking patterns (refresh rate of $10 \mathrm{~Hz}$ ) were presented to the other eye, on either side of fixation. Immediately after, a dot appeared at the location preceded by the left or right face and observers were required to report its location as quickly and accurately as possible.

a)

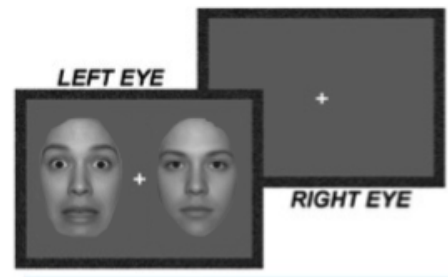

$500 \mathrm{~ms}$

b)

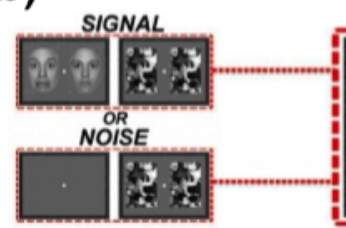

c)

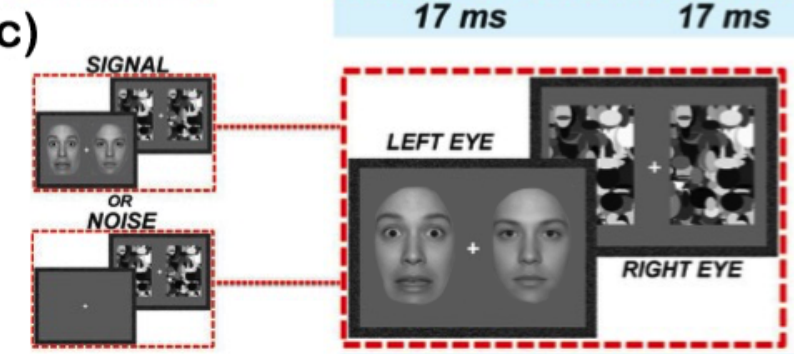

$500 \mathrm{~ms}$

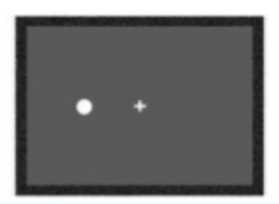

Until response

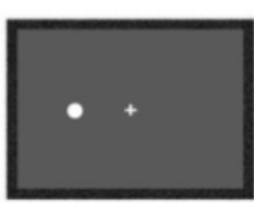

Until response

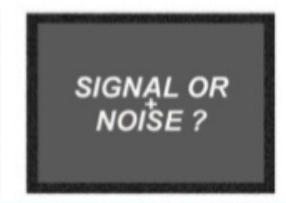

Until response

SIGNAL OR

NOISE?

Until response

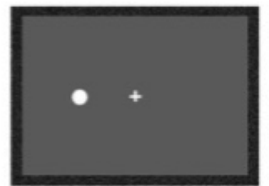

SIGNAL OR

NOISE?

Until response 
EMOTION AND ATTENTION

271 Figure 2. Schematic of trial sequences for the three presentation conditions. a) standard

272 presentation b) masked presentation c) CFS presentation. Masked and CFS trials had an equal

273

274

275 number of signal trials (trials where face stimuli were presented) and noise trials (trials where no face stimuli were presented) - these are shown in the leftmost panels.

\section{Stimulus Pairing Conditions}

Within each presentation condition (standard, masked, CFS) there were two stimuluspairing conditions, corresponding to emotion bias trials, and face bias trials (see Figure 3).

\section{Emotion bias}

Mirroring conventional visual probe studies, emotion bias trials (64 trials), were designed to measure whether an emotion bias exists, i.e. a tendency to allocate attention to emotional stimuli when a neutral and an emotional stimulus compete for resources (Figure 3a). The face presentation consisted of an emotional face ( 32 fear, 32 happy) presented to one side of fixation and a neutral face presented to the other. Within each emotion bias pair, half of the trials were valid (subsequent probe appeared in the location of the emotional face) and half were invalid (probe appeared in the location of neutral face). These trials were repeated with face stimuli presented in both normal (16 trials, figure 3a) and upside-down negative configurations (16 trials, figure $3 b)$.

\section{Face bias}

Face bias trials (48 trials) were designed to measure any bias for more face-like stimuli in the allocation of selective attention when normal and upside-down negative face stimuli (with matching emotional expression) compete for resources (Figure 3c). In face bias trials, a normal face (16 neutral, 16 fearful, 16 happy) was presented on one side of fixation and a face with the same emotional expression, but in an upside-down negative configuration 


\section{EMOTION AND ATTENTION}

was presented on the other. Within each face bias paring, half of the trials were valid

297 (subsequent probe appeared in the location of the normal face) and half were invalid (probe appeared in the location of upside-down negative face).

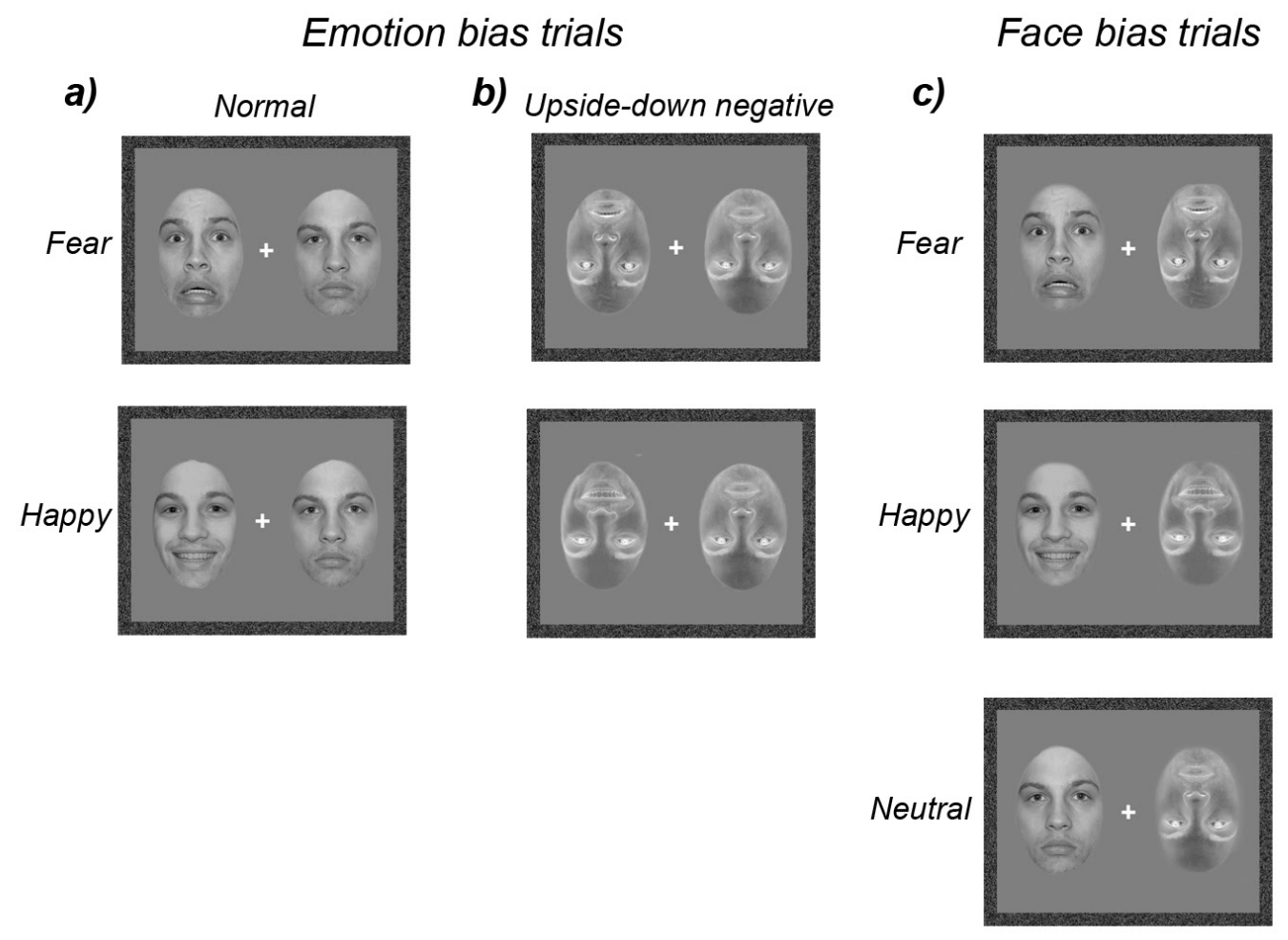

299

300 Figure 3. Schematic examples of each stimulus pairing condition. a) Emotion bias trials

301 (normal configuration). A normal emotional (fear or happy) and normal neutral face were

302 presented either side of fixation. b) Emotion-bias trials (upside-down negative configuration).

303 An upside-down negative emotional (fear or happy) and upside-down negative neutral face were presented either side of fixation. c) Face bias trials: a normal (fear, happy or neutral) and upside-down negative face (same expression) stimulus were presented either side of fixation.

$50 \%$ of the trials within the CFS and masked presentation conditions (112 masked, 
EMOTION AND ATTENTION

311 were presented prior to the mask (for masked presentations) or to the opposite eye to the mask (for CFS presentations). If observers are unaware of the stimuli, they should perform at

313 chance in discriminating signal trials from noise trials (Wiens, 2008). Thus, on each trial,

314 after the observer reported the location of the probe, they were prompted to indicate whether

315 the preceding presentation had been a 'noise' trial or a 'signal' trial (by pressing the up or

316 down arrow key). It was clearly explained to the participants that, within those trials that

317 contained a mask, faces were presented on only $50 \%$, and that they had to discriminate these

318 cases from those in which no faces were presented. Participants were also informed that there

319 were no time constraints for this response and that they should prioritise accuracy over speed.

320 The 224 trials for each presentation condition meant that this forced choice task had adequate

$321(80 \%)$ power to detect even very small deviations from chance performance (Cohens $h$ of

3220.16 or larger).

323 Summary

All 41 observers completed 336 signal trials - 112 trials for each of the 3 presentation conditions (standard, masked, CFS), each comprising (i) 64 emotion bias trials: 2 emotions (fear vs. neutral, happy vs. neutral) x 2 face configurations (normal, upside-down negative) $\mathrm{x}$ 16 repetitions, and (ii) 48 face bias trials: 3 emotions (neutral, fear, happy) x 16 repetitions). Participants also completed 224 noise trials (112 masked, 112 CFS). The side of the emotional /upside-down negative face, the eye of face presentation, the location of the probe and the validity of the probe were counterbalanced. Trial order was randomized for each participant. 
EMOTION AND ATTENTION

335 Following standard practice, $d$ ' values were computed from the difference between

336 the z-transformed hit rates (proportion of signal trials that were correctly identified) and false

337 alarm rates (proportion of noise trials that were incorrectly classified as signal trials). For

338 masked presentations, $d$ ' was consistent with poor discrimination between signal and noise

339 trials - at the group level, performance was not significantly better than chance $(M=0.04, t$

$340(40)=1.54, p=.130)$. No individual observer significantly exceeded chance performance in

341 correctly discriminating signal and noise trials (assessed via binomial test, upper binomial

342 limit $=127$ correct responses). For CFS presentations, performance was slightly higher and

343 significantly different from zero at the group level $(M=0.06, t(40)=2.55, p=.015)$. At the

344 individual level, two observers performed significantly above chance in distinguishing signal

345 and noise trials. These two observers were excluded from further analyses (with the

346 exception of the correlation analyses shown in Figure 6). After removal of these observers,

347 the group $d$ ' was not significantly different from zero for either masked $(M=0.04, t(38)=$

$3481.44, p=.158)$ or CFS $(M=0.04, t(38)=2.01, p=.051)$ presentations.

$349 \quad$ Visual Probe Data

350

Data reduction and global measures.

351

Preliminary inspection of the data revealed that one observer only achieved 52\%

352

accuracy in the probe discrimination task. Given the trivial difficulty of this task

(discriminating left probes from right probes) we reasoned that this observer did not engage

with the task requirements and thus their data were not analysed further. The remaining observers achieved near- ceiling accuracy $(M=98.37 \%, S D=1.97 \%$. Response times (RTs) corresponding to incorrect responses were removed ( $0.75 \%$ of RT data) and a log transform was applied to correct for skew. The mean $\log \mathrm{RT}$ was calculated for each observer for each presentation condition and cue validity. Values that were more than 3 standard deviations

359 from these means were defined as outliers and removed (1.53\%). The analyses reported 
EMOTION AND ATTENTION

360 below were conducted on the remaining $97.72 \%$ of the RT data. After the removal of outliers,

361 RTs were within the normal range for visual probe studies $(M=387 \mathrm{~ms}, S D=101 \mathrm{~ms})$.

362

363

364

365

366

367

368

369

370

371

372

373

374

375

376

377

378

379

380

381

382

383

384

\section{Emotion bias.}

To test whether observers' attention was drawn to emotional faces, we calculated an emotion bias score from the emotion bias trials (invalid RT - valid RT) for each stimulus condition (fear or happy, within normal or upside-down negative configurations) such that positive values indicate that attention is drawn to the location of the emotional (rather than neutral) expression. These are summarised in Figure 4a. In addition, we calculated the overall emotion bias in each of the three presentation conditions (pooled across expression and configuration), these are shown in Figure $4 \mathrm{~b}$.

An overall emotion bias was detected for standard presentations $(M=19.79 \mathrm{~ms}, t$ $(37)=2.33, p=.025)$, corresponding to a modest effect size $\left(d_{z}=0.38,95 \%\right.$ CI $\left.[0.050 .71]\right)$. However, in the masked and CFS conditions, no overall emotion bias was detected (masking: $M=-7.26 \mathrm{~ms}, t(37)=-0.87, p=.382$, CFS: $M=0.07 \mathrm{~ms}, t(37)=0.01 p=.991$ ), and the effect sizes were small (masking: $d_{z}=-0.14,95 \%$ CI [-0.46 0.17], CFS: $d_{z}=0.00$, $95 \%$ CI [ $-0.320 .32])$. When comparing the magnitude of the emotion bias across conditions, a main effect of presentation condition was detected $(F(2,74)=3.096, p$ $=.046)$. Thus, our data suggest that observers' attention was drawn towards emotional stimuli under standard presentation, in which stimuli were fully visible, but not in masking or CFS trials.

We can consider whether the emotion bias was modulated by expression (fear vs. happy) or configuration (normal vs. upside-down negative); if attentional allocation was driven by affective content, (i.e. the meaning of the stimuli) then we expect a larger emotion bias in the normal than the upside-down negative configuration, because expressions are harder to discriminate in the upside-down negative configuration. There 


\section{EMOTION AND ATTENTION}

was no significant interaction between expression and stimulus configuration in any presentation condition (2-way ANOVAs, all $p$-values $>.45$ ). Importantly, this suggests

387 that facial expression had no effect on attentional allocation beyond that explained by basic low-level variability between expressions. In fact, the emotion bias in the standard presentation condition (widely reported in previous literature: Bar-Haim et al., 2007) was smaller for normal than upside-down negative stimuli (normal: $M=18.56$, upside-down negative: $M=21.02, t(37)=-0.17, p=.863)$.

a)

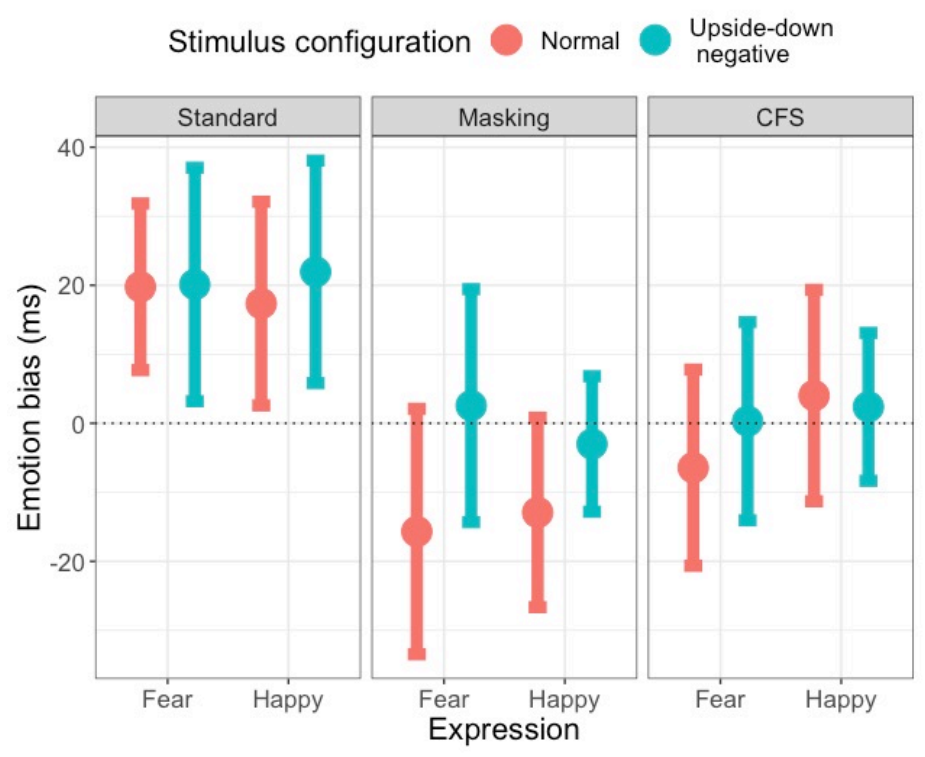

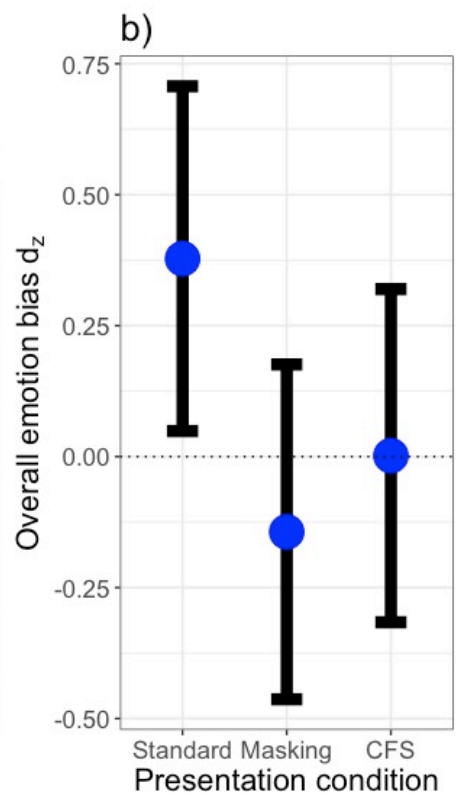

392

393

394

395

Figure 4. Attentional biases in emotion bias trials. a) Emotion bias (invalid RT - valid RT) plotted as a function of expression, stimulus configuration (normal, upside-down negative) and presentation condition. Error bars are +/- 1 SE. b) The overall emotion bias, expressed as Cohen's $d_{z}$ is plotted as a function of presentation condition. Error bars are $95 \%$ confidence intervals.

Face bias. 
EMOTION AND ATTENTION

400

401

402

403

404

405

406

407

408

409

410

411

412

413

414

415

416

417

418

419

420

421

422

423

424

To determine whether observers' attention was directed to more face-like stimuli, i.e.

normal faces, as opposed to upside-down negative faces, we calculated a face bias score from face bias trials (invalid RT - valid RT) for each stimulus condition, such that positive values indicate that attention is drawn to the location of the normal face. The resultant face biases are summarised in figure 5a. Figure $5 \mathrm{~b}$ shows the overall face bias effect size in each presentation condition (pooled across all stimuli). We detected a significant face bias in standard trials $(M=18.33 \mathrm{~ms}, t(37)=2.19, d z=0.36,[0.030 .68], p=.035)$ and in CFS trials $(M=28.39 \mathrm{~ms}, t(37)=2.98, d z=0.49,[0.150 .82], p=.005)$ but not in masking trials $(M=$ $11.01 \mathrm{~ms}, t(37)=-1.48, d z=-0.24,[-0.560 .08], p=.146)$. A significant effect of presentation condition $(F(2,74)=5.64 p=.005)$ was detected. Post hoc tests revealed that both standard $(p=.015)$ and CFS $(p=.003)$ presentations yielded larger face biases than masked presentations. We can ask whether these biases towards normal faces are modulated by expression. However, there was no significant main effect of expression $F(2,74)=.099, p$ $=.906$, or interaction with presentation condition $F(4,148)=.92, p=.455$. We detected no effect of expression within any presentation condition (one-way ANOVAs, all $p$-values $>$ .377). Despite observers indicating low levels of overall sensitivity to the presence $v$ absence of stimuli, one possibility is that small differences in sensitivity to normal vs. upside-down negative faces (e.g. Jiang, Costello \& He, 2007) could account for the face biases in CFS trials. Since both normal and upside-down negative stimuli are presented in face bias trials, we tested this possibility by using the signal detection data from emotion bias trials task to predict face bias. Since 'noise' trials only differ at the level of presentation condition (CFS, masked), it is not possible to calculate $d$ ' seperately for individual trial types (e.g. normal and upside-down negative trials). For this reason, we used hit rate as our measure of sensitivity in this analysis. We detected no association between differential hit rate (normal - upside-down negative hit rate) and face-bias magnitude $(r(36)=-.06,[-.38 .261], p=.703$. 
EMOTION AND ATTENTION

a)

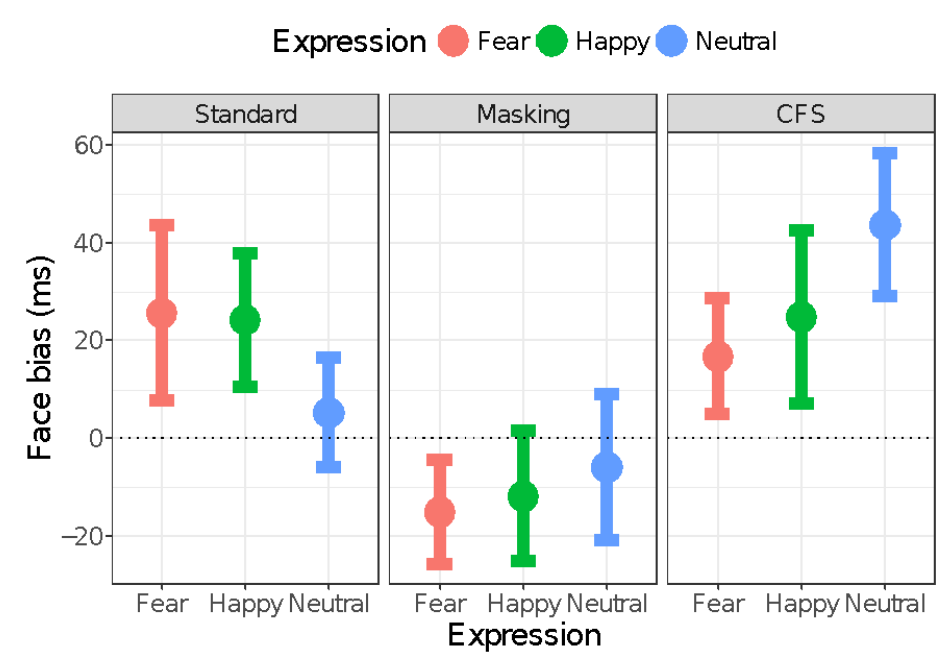

b)

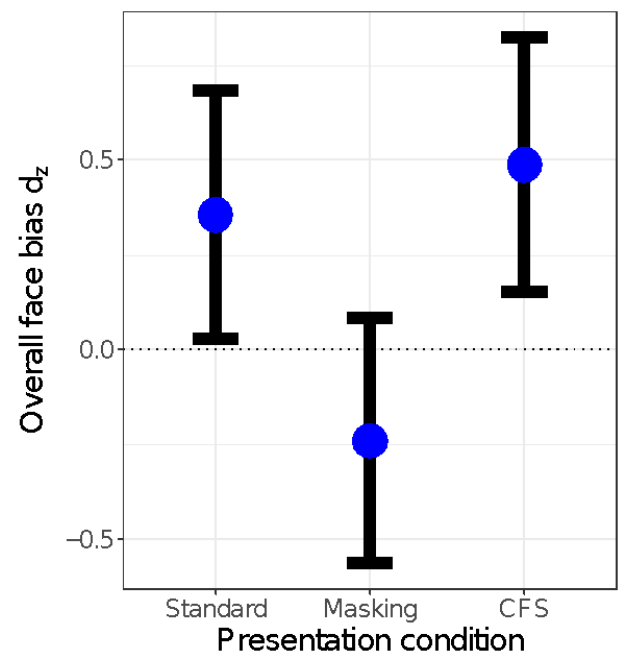

425

426

427

428

429

430

431

432

433

434

435

436

437

438

439

440

441

Figure 5. Attentional biases in face bias trials. a) Face bias scores (invalid RT - valid RT)

plotted as a function of expression and presentation condition. Error bars are +/- 1 SE. b) The overall face bias, expressed as Cohen's $\mathrm{d}_{\mathrm{z}}$, as a function of presentation condition. Error bars are $95 \%$ confidence intervals.

\section{Modelling of Visual Probe Data}

To better understand the stimulus attributes that predict attentional capture, and their relative importance, we developed a simple model to explain not just the mean difference between valid and invalid RTs within each stimulus pairing (which discards some, potentially informative RT information) but the full set of raw response times. In the visual probe paradigm, each trial represents a competition for attention between two stimuli (A and B).

We use the term 'salience' (S) to represent the capacity of each stimulus to capture attention, i.e. $S_{A}$ and $S_{B}$. If $S_{A}>S_{B}$ then, when a probe follows stimulus $A$, reaction times $\left(R_{A}\right)$ will be shorter, on average, than when it follows stimulus $\mathrm{B}\left(\mathrm{RT}_{\mathrm{B}}\right)$. Reaction times are well approximated by a log-normal distribution, and so we model the probability distribution of reaction times as: 
EMOTION AND ATTENTION

442

$$
p\left(\log \left(R T_{A}\right)=t\right)=\frac{1}{\sqrt{2 \pi \sigma^{2}}} e \frac{\left(t-\mu-S_{A}+S_{B}\right)}{2 \sigma^{2}}
$$

443

444

445

446

447

448

449

450

451

452

453

454

455

456

457

458

459

460

461

462

463

464

465

where $\mu$ and $\sigma$ give the mean and standard deviation of the baseline distribution of $\log (\mathrm{RT})$, i.e. with two equally salient stimuli.

We considered different stimulus attributes that might modulate stimulus salience.

These included emotional content (emotional vs. neutral), threat (the additional salience of fearful faces, relative to happy faces) and configuration, i.e. similarity to a normal face (normal vs. upside-down negative). We tested models that included only additive (e.g. emotion + configuration) or also interactive (e.g. emotion $*$ configuration) combinations of these variables. These parameters were used to define the relative salience of stimuli, where a neural upright face is given a nominal saliency of 0 . For example, if the salience of a stimulus were independently predicted by emotion and configuration (Model 5, Table 1), then the (relative) salience of normal fearful and happy faces is given by the emotion coefficient, and the salience of neutral upside-down negative faces is given by the configuration coefficient. A positive emotion coefficient indicates that emotional (fear and happy) faces are more salient than neutral faces and a negative configuration coefficient indicates that normal faces are more salient than upside-down negative faces. Interaction terms allow us to model the situation in which the effect of emotion or expression differs between normal and upsidedown negative configurations. 
EMOTION AND ATTENTION

467 Table 1.

468

Summary of Tested Models and their Parameters

\begin{tabular}{ll}
\hline Model & Parameters \\
\hline 1 & (Null model) \\
2 & configuration \\
3 & emotion \\
4 & emotion, threat \\
5 & configuration, emotion \\
6 & configuration, emotion, threat \\
7 & configuration, emotion, configuration*emotion \\
8 & configuration, emotion, threat, config*emotion, config*threat.
\end{tabular}

Because our models differed in complexity (the number of free parameters), leave one out cross-validation (LOO xval) was used to evaluate the generalisation performance of all models and avoid over-fitting (see Supplementary Material S1). In this method, a model is fit to $N-1$ observers (training data) and the fitted values are used to predict the data from the 'left out' observer (test data). The performance of the model in predicting the new data (in terms of error) directly reflects the generalisation performance of the model in predicting new 'unseen' data. One appealing property of LOO xval is that, unlike model performance indices such as Bayesian Information Criterion (BIC) or Akaike's information Criterion (AIC), there is no need to apply (ad-hoc) criteria to determine whether a more complex model's improved fit (in terms of likelihood, or other goodness-of-fit metric) is justified by the increased number of free parameters. Instead, the LOO method will naturally reveal the number of parameters required to model the signal (but not the noise) within the dataset: Unnecessarily complex models are implicitly penalised by this procedure, since they 'overfit' to the training data and therefore will have lower performance in predicting the left out (test) data. The results of the LOO xval procedure are displayed in Supplementary Material S2 (this figure illustrates that several models perform worse at cross-validation than the null model, 
EMOTION AND ATTENTION

486 demonstrating the penalty associated with over-fitting). The fitted parameters for all models

487 are summarised in Supplementary Material S3.

488 The model that best explains stimulus salience, and thus participants' RTs, varied

489 according to presentation condition (see Table 2). Under standard presentations, where

490 observers were fully aware of the face stimuli, the data were best explained by a model of

491 salience that included both emotion and threat (model 4). In other words, emotional stimuli

492 attracted attention more effectively than neutral stimuli, and this effect was increased for

493 threat-relevant fear faces. Importantly, models involving interactions between configuration

494 and emotion or threat did not improve on this model. In other words, under conditions of full

495 awareness, there is no evidence that emotional stimuli have increased salience, beyond that

496 determined by their low-level image properties. The modelling results are broadly in line

497 with the traditional visual-probe analyses reported above, although they additionally have the

498 increased sensitivity to reveal the increased salience of fear, relative to happy faces.

500 Table 2.

501 Best Fitting Models for Each Presentation Condition

\begin{tabular}{lll}
\hline Presentation Condition & Best Model & Fitted coefficients $M(S D)$ \\
\hline Standard & 4 & emotion: $5.98(0.56)$ \\
& & threat: $2.14(0.64)$ \\
Masking & 2 & configuration: $5.31(0.42)$ \\
CFS & 2 & configuration: $-6.02(0.37)$ \\
\hline
\end{tabular}

502

For both masked and CFS presentations, the best model of participants' RTs included

504 only the stimulus configuration (model 2). More complex models, involving stimulus

505 emotion or threat did not have greater cross validation performance than the null model. This, 
EMOTION AND ATTENTION

506 alongside the previous analyses, suggests that participants' responses are not affected by

507 facial emotion when faces are presented outside of awareness via backward masking or CFS.

508

509

\section{Association With Awareness and Anxiety Measures}

510

Our previous work (Hedger et al., 2016) suggests that some emotion-related biases

511 found in backward masking and CFS paradigms may be due to, or modulated by observers'

512 awareness of the stimuli. To determine whether such an effect exists in the current data, we

513 examined the relationship between attentional bias and awareness of the stimuli (as indexed

514 by performance in the 'signal' vs. 'noise' discrimination task) in the masked and CFS

515 presentation conditions. For each observer, we computed a single attentional bias score,

516 collapsed across all stimulus types, and a single $d$ ' score for stimulus awareness, collapsed

517 across CFS and masked presentations.

518 Performance in the awareness task (i.e. the ability to distinguish 'signal' from 'noise'

519 trials) was significantly and positively correlated with attentional bias $\left(F(1,38)=4.693, R^{2}=\right.$

$520.086, p=.037$ ), as shown in Figure 6a, suggesting that attentional biases are inflated when

521 observers have some awareness of the stimuli. Notably, the best-fit line passes very close to

$522(0,0)$, suggesting that awareness of the stimuli not only increases attentional bias, but may be

523 required for attentional bias effects to occur. When the data were split by stimulus pairing,

524 this revealed that the association between awareness and attentional bias was mostly driven

525 by the emotion bias $F(1,38)=3.311, R^{2}=.080, p=.077$ (Figure 6b) and that the face bias

526 had a weaker association with awareness $F(1,38)=2.003, R^{2}=.025, p=.166$ (Figure 6c). 
EMOTION AND ATTENTION

a)

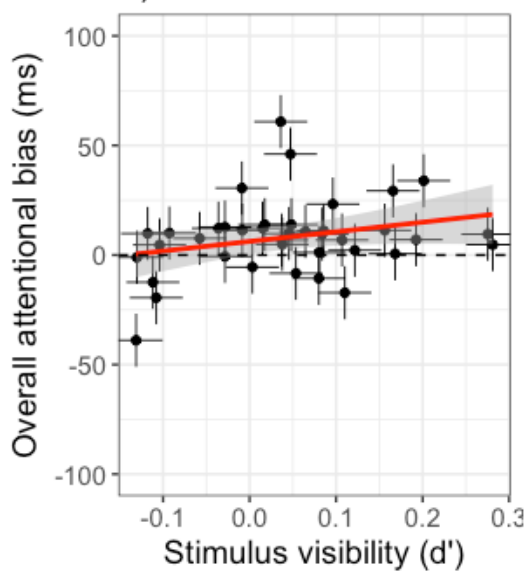

b)

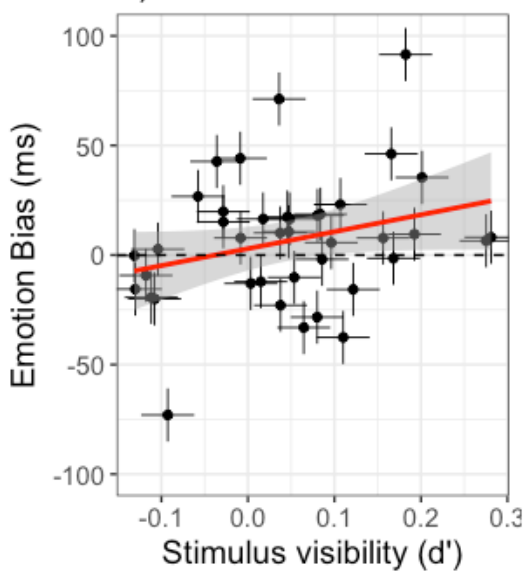

c)

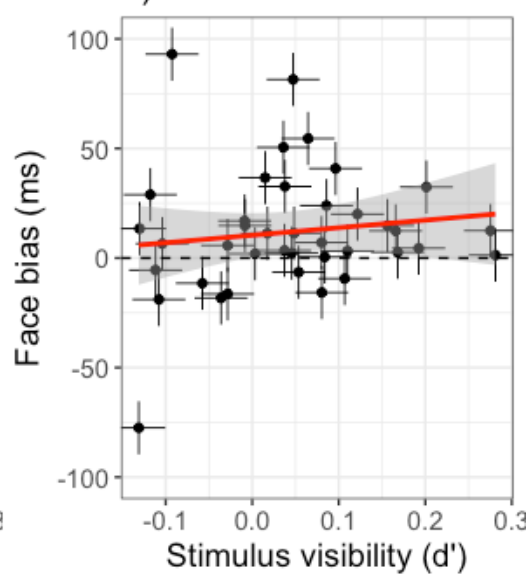

Figure 6. a) Association between overall $d$ ' and overall attentional bias score. b) Association

531 between $d^{\prime}$ ' and emotion bias score. c) Association between $d$ ' and face bias score. Red lines

532 are the least squares fit to the data, shaded region is +/- $1 \mathrm{SE}$.

533

As shown in supplementary material S4, our measures of anxiety (STA-T, SPI, SIAS) were highly correlated (all Pearson's' $r>.43$ all ps $<.01$ ), indicating good reliability of these measurements. Our primary research questions were unrelated to individual differences (e.g. participants' levels of anxiety). However, previous research has suggested that biases toward emotional stimuli are inflated amongst anxious observers (Bar- Haim et al, 2006). For this reason, we performed correlation analyses to examine the association between attentional bias and anxiety measures (STAI-T, SPS, SIAS) in each presentation condition. No correlations were detected in any presentation condition for either emotion or face bias scores

542 (all $p$-values $>.05$ ). These correlations and their associated confidence intervals can be found 543 in supplementary material S5. 
EMOTION AND ATTENTION

546 Our experimental design allowed us to assess attentional orienting to neutral and

547 emotional face stimuli under different conditions of awareness. A few key findings emerged:

548 (i) In the standard, supraliminal paradigm, we found evidence that emotional faces attract

549 attention when competing with neutral faces. Our stimulus configuration manipulation

550 (normal $\mathrm{v}$ upside-down negative) allowed us to determine the extent to which this is driven

551 by the low-level image properties of the stimuli vs. their recognisable emotional content. In

552 fact, the effect of emotion was slightly larger for upside-down negative faces, suggesting that

553 attentional allocation within our visual probe task was not driven by recognisable emotion.

554 No emotion biases were found when stimuli were presented outside of awareness via

555 masking or CFS. (ii) Normal faces attracted attention over upside-down negative faces within

556 the standard and CFS conditions, suggesting a preference for more natural, face-like stimuli.

557 (iii) Attentional effects were predicted by observers' awareness of the stimuli, suggesting that

558 attentional biases are modulated, or even driven by awareness.

$559 \quad$ Attentional Capture by Emotionally Salient Stimuli

$560 \quad$ For standard, 500ms supraliminal trials, we observed attentional biases towards

561 emotionally salient stimuli. Importantly, these effects were not reduced when the stimuli

562 were presented in the upside-down negative condition (in fact, they increased slightly). This

563 suggests that the apparent effect of emotion on attentional allocation within the standard

564 (conscious) visual probe paradigm was driven by low-level stimulus factors (e.g. luminance

565 contrast), rather than emotional valence per se; the upside-down negative faces have vastly

566 reduced recognisable emotional content (Gray et al., 2013). This finding suggests that

567 previous reports of an attentional bias towards emotional stimuli may be attributable, at least

568 in part, to low-level differences between stimuli. Fearful and happy facial expressions in

569 particular tend to have more contrast energy at the spatial scales humans are sensitive to, 
EMOTION AND ATTENTION

570 relative to neutral faces (Hedger et al., 2015). Thus, a processing advantage for these

571

572

573

574

575

576

577

578

579

580

581

582

583

584

585

586

587

588

589

590

591

592

593

594

expressions is predicted on the basis of simple sensory factors alone.

When stimuli were suppressed from awareness via masking and CFS, emotion bias effects were small and insignificant. However, as with any null result, it is worth discussing possible sources of a type 2 error. On a statistical level, it is important to note that power was high and the sample size calculations were based on a large body of previous literature (see 'participants' section). Secondly, in relation to the sample characteristics, the mean trait anxiety level was relatively high $(M=41.46)$ and above the value expected to produce detectable biases towards threatening stimuli under subliminal presentations (Hedger et al., 2016). Unlike studies that have solely used masking to manipulate awareness, it is unlikely that null effects in unconscious presentations can be explained by simple restrictions on presentation time, since this was equated in normal and CFS trials (500 ms). Importantly, we detected significant attentional bias effects under standard presentation conditions, suggesting that the task, in itself, was a sensitive measure of attentional allocation. Our results are consistent with studies that have failed to detect evidence of emotional modulation of attention under masking (Fox, Cahill, \& Zougkou, 2010; Koster et al., 2007) and CFS (Hedger, Adams, \& Garner, 2015a).

\section{The Thorny Issue of Low-level Confounds}

In our study, we observed equivalent attentional cuing effects for normal and upsidedown negative stimuli and thus concluded that variability between stimuli in low-level image properties drives attentional cuing. It is worth discussing some competing explanations for our findings.

First, we consider whether normal and upside-down negative stimuli share some property (other than luminance contrast) that drives the attentional effect. For instance, upside-down negative stimuli could retain some (reduced) recognisable emotional content, 
EMOTION AND ATTENTION and this attracts attention to the same extent as the emotion as content within normal stimuli.

We think this is unlikely for two reasons. Firstly, upside-down negative stimuli actually give rise to slightly larger attentional effects than normal stimuli (rather than smaller effects, see Figure 4). Secondly, we have previously demonstrated that upside-down negative facial expressions are not evaluated as being significantly different from neutral valence according to an Extrinsic Affective Simon Task (EAST). Specifically, the disruption caused by this manipulation was found to not simply reduce the magnitude of valence judgements, but generated a qualitatively pattern of effects (see Gray et al., 2013 experiment 2).

A second possibility is that the similar effects observed for normal and upside-down negative stimuli are not driven by a single, shared mechanism, but by two different mechanisms that happen to produce effects of similar magnitude. For example, might it be that normal faces capture attention via their emotional content, whereas upside-down negative faces capture attention via their low-level image properties? Again, we believe that this explanation is unlikely. Firstly, it contradicts standard experimental logic - if one manipulates a potentially important experimental variable (e.g. emotional content), and finds no effect on the dependent variable (e.g. attentional allocation), the standard conclusion is that the variable is not as important as previously hypothesised. Second, we have previously demonstrated that stimulus detection (across both normal and upside-down negative faces) is much better predicted by variations in luminance contrast than by variations in perceived valence or arousal (Hedger et al 2015b experiment 2). Moreover, a quantitative analysis of previous literature also reveals that the affective content of a stimulus is a poor predictor of its ability to capture attention in the MVP paradigm: our meta-analysis revealed that fearful faces are the only class of threat stimuli that reliably generate threat-related biases in the MVP paradigm. Angry faces, in contrast, yield small, non-significant effects (Hedger et al., 2016). Angry faces signal a direct threat to the observer, whereas fearful faces indicate the 
EMOTION AND ATTENTION

620 presence of a proximal threat. If threat directs attention within VP paradigms, this should be

621

622

623

624

625

626

627

628

629

630

631

632

633

634

635

636

637

638

639

640

641

642

643

644 apparent for indicators of proximal threat (i.e. fearful faces), and direct threat (i.e. angry faces). Instead, parsimony favours an account based on the sensory advantages of the fear expression over the neutral or angry expression (Hedger et al., 2015b, Lee et al., 2013).

Another potential objection could be that the unfamiliar/unusual quality of upside-down negative faces might drive attention. Crucially, it is worth noting that this concern would not apply to emotion bias trials, since both emotional and neutral face stimuli were presented in the upside-down negative configuration on these trials. We also explicitly test for the possibility that 'unfamiliar' upside-down negative stimuli attract attention over the more 'familiar' normal stimuli in face-bias trials. However, the opposite effect was observed: attention was instead drawn to faces in the normal configuration. Moreover, rendering our upside-down negative stimuli 'unfamiliar' is a necessary and desired effect of the manipulation - if the face stimuli were recognisable in the upside-down negative condition, they would not provide a valid control for variations in low-level image properties.

Other objections to the conclusions drawn from our upside-down negative manipulation are more philosophical. Some authors have proposed that low-level image properties may drive efficient detection precisely because of their emotionality (Frischen, Eastwood, \& Smilek, 2008). Under this line of reasoning, labelling the low-level variability between stimuli as a 'confound' is problematic, because the communicated emotion is defined by its low-level properties (e.g. patches of high contrast signal fear). This would undermine the idea of attempting to control for low-level stimulus properties in any perceptual experiment. Like others (e.g. Becker, Anderson, Mortensen, Neufeld, \& Neel, 2011), we believe that this position is unfalsifiable and unsound. Clearly, not all high-contrast stimuli are fear-inducing, and as discussed above, not all threatening stimuli are highcontrast. Further, if one group of researchers holds the view that fear faces attract attention 
EMOTION AND ATTENTION

645 because of their threat relevance and another claims that fearful faces attract attention due to

646 low-level image properties, then the debate can only move forward by designing experiments

647 that attempt to resolve between these competing possibilities.

648 Considering all evidence, we believe that simple low-level variability between stimuli

649 provides the most parsimonious account of the attentional effects that we observe. Other

650 explanations are either i) harder to support with the available data, or ii) require additional

651 assumptions.

652 Implications for Clinical Work.

653 Failing to control for, or characterize low-level stimulus properties can have serious

654 implications. Consider populations who might be expected to show diminished threat

655 processing, such as patients with a recent brain injury (Tsuchiya et al., 2009), or individuals

656 who have received an intervention to alleviate anxiety symptoms (Murphy, Downham,

657 Cowen, \& Harmer, 2008). An apparent threat-related bias in these populations may be

658 wrongly as interpreted as being indicative of 'unimpaired threat processing' or a 'failed

659 intervention'. In reality, it may be that these observers simply have normal sensitivity to the

660 low-level variability between neutral and emotional stimuli. Indeed, much of the

661 unaccounted-for variation in the efficacy in a behavioral intervention for anxiety such as

662 attentional bias modification (Mogg \& Bradley, 2018) could be explained by low-level

663 variability between stimulus categories.

664 Attentional Preference for 'Face Like' Stimuli

665 We observed evidence for attentional biases toward normal faces when competing

666 with manipulated, upside-down negative faces in both standard and CFS trials. The latter

667 finding is consistent with a large body of work from the breaking continuous flash

668 suppression (bCFS) literature, which has consistently demonstrated that upright faces break

669 CFS suppression faster / more frequently than inverted faces (Jiang, Costello, \& He, 2007; 
EMOTION AND ATTENTION

670 Stein \& Sterzer, 2012). In addition, there is evidence from fMRI that face-selective regions of

671 the temporal cortex are differentially activated by upright vs. inverted faces even when these

672 are presented under CFS (Jiang \& He, 2006). The present study extends this literature by

673 providing the first behavioural evidence that face-like stimuli attract spatial attention under

674 CFS. Based on the present data, inferring a causal link between preferential detection of

675 upright, normal faces (in bCFS studies) and attention to normal faces (in the present study)

676 would be premature. Future work could test for the presence and directionality of such a

677 causal relationship.

678

Why is it that CFS seems to spare selective attention to face like configurations (in

679 face bias trials), but not emotional expressions (in emotion bias trials)? One possibility is that

680 discriminating a face from a non-face (a coarse, basic-level classification) is easier than

681 discriminating different expressions (a finer, sub-ordinate classification) and may thus be less

682 affected by degradation associated with CFS suppression. This sensitivity to upright faces cannot be explained by low-level stimulus properties such as contrast and spatial frequency profile, which are preserved after spatial and contrast inversion. Instead, the prioritised detection of upright faces appears to reflect some higher-level 'face-sensitive' process. An alternative explanation is that the preferential processing of upright faces does not reflect face-sensitive processes, but rather the fact that 'top heavy' patterns in general are more easily detectable, since humans have a robust upper hemifield advantage in basic visual sensitivity (Skrandies, 1987). In fact, recent work has shown that upright 'protofacial' stimuli

690 (a simple triangular configuration of dots, resembling the position of the eyes and mouth) break CFS more rapidly than their inverted counterparts (Akechi et al., 2015). effects driven by simple differences in sensitivity in the upper and lower hemifield. Clearly, these two possibilities have drastically different implications for the level and complexity of 
EMOTION AND ATTENTION

695 visual processing that transpires without awareness. This, in turn, suggests caution when

inferring high level processing based on a preference for normal, upright face configurations.

697

698

699

700

701

702

703

704

705

706

707

708

709

710

711

712

713

714

715

716

717

718

719

\section{Implications for Paradigms used to Manipulate Awareness}

A recent concern, that has been raised by many, is whether the perceptual suppression induced by techniques such as masking and CFS are functionally similar to those that may occur under natural viewing conditions (Blake, Brascamp, \& Heeger, 2014; Hesselmann \& Moors, 2015). If they are not, then studies employing these techniques may tell us about the peculiarities of the techniques used, rather than revealing any characteristics of unconscious processing that generalise to natural viewing conditions. A related concern is that conclusions emanating from different paradigms used to manipulate awareness may not generalise to one another, since they do not index the same level of unconscious processing (Breitmeyer, 2015; Dubois \& Faivre, 2014). This entails that a null effect in one paradigm does not necessarily entail the absence of unconscious processing, since affirmative findings may be found with a different paradigm. Our findings strengthen these concerns. For instance, based on our data from the standard and masked presentations alone, one could conclude that an attentional preference for face-like configurations (in face bias trials) depends on their conscious registration. By contrast, when these data are considered alongside the CFS data, one could conclude that the absence of such effects may be due to the methodological limitations of the masking paradigm. For instance, it may be the case that face-sensitive processes simply require a more sustained and robust visual signal than is supported by very brief, masked presentation. Similarly, the absence of an emotion bias in CFS or masked presentations does not necessarily imply that emotional stimuli fail to modulate attention under all conditions of unawareness. For instance, Faivre, Berthet and Koudier (2012) found that affective priming was eliminated when primes were presented under CFS, but robust priming effects were observed when primes were rendered indiscriminable by crowding. The study of unconscious 
EMOTION AND ATTENTION

720 processing is thus highly susceptible to the error of 'denying the antecedent' when

721

722

723

724

725

726

727

728

729

730

731

732

733

734

735

736

737

738

739

740

741

742

743

744 interpreting null effects.

\section{Implications for Assessment of Awareness}

If attentional cuing operates independently of awareness of the cuing stimuli, we

should expect no association between discrimination of stimulus presence and the magnitude of the attentional cuing effect. Instead, our data reveal that increased stimulus awareness (as assessed by $d$ ') predicted increased attentional biases, despite the limited range of $d$ ' values and our sample's relatively low level of sensitivity. Recent research employing stringent signal detection measures of awareness have revealed that observers are more capable than previously assumed at detecting brief, masked signals. In fact, one study has shown that the majority of observers can reliably detect images of fearful faces that are masked after 25 , or even 17 ms (Szczepanowski \& Pessoa, 2007). Although these deviations from chance performance were small, they are non-trivial in the context of the attentional effects emanating from the masked visual probe paradigm, which are also very small. This, taken together with our own data, illustrates the importance of providing sensitive, well-powered and objective awareness measures.

\section{Implications for Emotion Theory}

Several dominant neurocognitive theories of emotion assume independence of affective processing and awareness. Various 'dual pathway' models rest on the assumption that processing of affective visual stimuli involves a separable sub-cortical visual pathway that bypasses the visual cortex and projects affective information rapidly to emotionallyresponsive structures (e.g. the amygdala) independently of awareness. The first explicit model of this kind was formulated as early as 1885 (Lange, 1885) and adaptations of this idea have been presented more recently (LeDoux, 1996; Tamietto and de Gelder, 2010). Clearly, it would be rash to challenge the neuroanatomical aspects of such theories on the basis of our 
EMOTION AND ATTENTION

745 behavioural data. However, regardless of whether such a pathway exists, we find no

746 evidence that it supports the preferential processing of threat stimuli in the absence of

747 awareness. This accords with recent suggestions that the proposed subcortical pathway is

748 highly unlikely to have the computational properties required to perform processes such as

749 object identification, which would be required in order to differentiate threatening from

750 nonthreatening signals (Cauchoix \& Crouzet, 2013).

751

$752 \quad$ Conclusion

753

In conclusion, our data suggest that attentional capture by emotionally salient stimuli

754 is predicted by awareness. We detected attentional cuing effects under normal viewing

755 conditions, but not under two different conditions of unawareness. Moreover, we provide

756 direct evidence that an observer's awareness of stimuli predicts the magnitude of attentional

757 cuing effects. Finally, even under full awareness, we found that attentional cuing by

758 emotionally salient stimuli was fully accounted for by low-level stimulus confounds. When

759 considered alongside our meta-analysis, these findings could motivate a reinterpretation of

760 previous literature and stimulate further well-controlled studies on the relationship between

761 emotion processing, attention and awareness.

762

763

764

765 
EMOTION AND ATTENTION

766

767

768

769

770

771

772

773

774

775

776

777

778

779

780

781

782

783

784

785

786

787

788

789

790

References

Akechi, H., Stein, T., Kikuchi, Y., Tojo, Y., Osanai, H., \& Hasegawa, T. (2015). Preferential awareness of protofacial stimuli in autism. Cognition, 143, 129-134. http://doi.org/10.1016/j.cognition.2015.06.016

Armony, J. L., \& Dolan, R. J. (2002). Modulation of spatial attention by fear-conditioned stimuli: an eventrelated fMRI study. Neuropsychologia, 40(7), 817-826.

Bar-Haim, Y., Lamy, D., Pergamin, L., Bakermans-Kranenburg, M. J., \& van IJzendoorn, M. H. (2007). Threat-related attentional bias in anxious and nonanxious individuals: a meta-analytic study. Psychological Bulletin, 133(1), 1-24. http://doi.org/10.1037/0033-2909.133.1.1

Becker, D. V., Anderson, U. S., Mortensen, C. R., Neufeld, S. L., \& Neel, R. (2011). The face in the crowd effect unconfounded: happy faces, not angry faces, are more efficiently detected in single- and multiple-target visual search tasks. Journal of Experimental Psychology. General, 140(4), 637-659.

Beaver, J. D., Mogg, K., \& Bradley, B. P. (2005). Emotional conditioning to masked stimuli and modulation of visuospatial attention. Emotion (Washington, D.C.), 5(1), 67-79. http://doi.org/10.1037/1528-3542.5.1.67

Blake, R., Brascamp, J., \& Heeger, D. J. (2014). Can binocular rivalry reveal neural correlates of consciousness? Philosophical Transactions of the Royal Society of London B: Biological Sciences, 369(1641), 20130211.

http://doi.org/10.1098/rstb.2013.0211

Breitmeyer, B. G. (2015). Psychophysical "blinding” methods reveal a functional hierarchy of unconscious visual processing. Consciousness and Cognition, 35, 234-250. https://doi.org/10.1016/j.concog.2015.01.012 
EMOTION AND ATTENTION

791 Carlson, J. M., \& Reinke, K. S. (2008). Masked fearful faces modulate the orienting of covert

792

793

794

795

796

797

798

799

800

801

802

803

804

805

806

807

808

809

810

811

812

813

814 Fox, E., Cahill, S., \& Zougkou, K. (2010). Preconscious processing biases predict emotional

815

816 spatial attention. Emotion (Washington, D.C.), 8(4), 522-529. http://doi.org/10.1037/a0012653

Carlson, J. M., Reinke, K. S., \& Habib, R. (2009). A left amygdala mediated network for rapid orienting to masked fearful faces. Neuropsychologia, 47(5), 1386-1389. http://doi.org/10.1016/j.neuropsychologia.2009.01.026

Cauchoix, M., \& Crouzet, S. M. (2013). How plausible is a subcortical account of rapid visual recognition? Frontiers in Human Neuroscience, 7, 39.

Chica, A. B., Martín-Arévalo, E., Botta, F., \& Lupiáñez, J. (2014). The Spatial Orienting paradigm: How to design and interpret spatial attention experiments. Neuroscience \& Biobehavioral Reviews, 40, 35-51. http://doi.org/10.1016/j.neubiorev.2014.01.002

Cohen, J. (1977). Statistical Power Analysis for the Behavioral Sciences. New York:

Routledge.

Dennett, D. C. (1993). Consciousness Explained. Penguin UK.

Dubois, J., \& Faivre, N. (2014). Invisible, but how? The depth of unconscious processing as inferred from different suppression techniques. Consciousness Research, 5, 1117. http://doi.org/10.3389/fpsyg.2014.01117

Faivre, N., Berthet, V., \& Kouider, S. (2012). Nonconscious influences from emotional faces: A comparison of visual crowding, masking, and continuous flash suppression. Frontiers in Psychology, 3, 129. http://dx .doi.org/10.3389/fpsyg.2012.00129

Fox, E. (2002). Processing emotional facial expressions: The role of anxiety and awareness. Cognitive, Affective \& Behavioral Neuroscience, 2(1), 52-63. reactivity to stress. Biological Psychiatry, 67(4), 371-377. http://doi.org/10.1016/j.biopsych.2009.11.018 
EMOTION AND ATTENTION

817 Frischen, A., Eastwood, J. D., \& Smilek, D. (2008). Visual search for faces with emotional

818

819

820

821

822

823

824

825

826

827

828

829

830

831

832

833

834

835

836

837

838

839

840

841 expressions. Psychological Bulletin, 134(5), 662-676.

Garrido, M. I. (2012). Brain Connectivity: The Feel of Blindsight. Current Biology, 22(15), R599-R600. http://doi.org/10.1016/j.cub.2012.06.012

Gray, K. L. H., Adams, W. J., Hedger, N., Newton, K. E., \& Garner, M. (2013). Faces and awareness: low-level, not emotional factors determine perceptual dominance. Emotion (Washington, D.C.), 13(3), 537-544. http://doi.org/10.1037/a0031403

Hedger, N., Adams, W. J., \& Garner, M. (2015a). Autonomic arousal and attentional orienting to visual threat are predicted by awareness. Journal of Experimental Psychology. Human Perception and Performance, 41(3), 798-806. http://doi.org/10.1037/xhp0000051

Hedger, N., Adams, W. J., \& Garner, M. (2015b). Fearful faces have a sensory advantage in the competition for awareness. Journal of Experimental Psychology. Human Perception and Performance, 41(6), 1748-1757. http://doi.org/10.1037/xhp0000127

Hedger, N., H, L., Garner, M., \& Adams, W. J. (2016). Are Visual Threats Prioritized Without Awareness? A Critical Review and Meta-Analysis Involving 3 Behavioral Paradigms and 2696 Observers. Psychological Bulletin, No Pagination Specified. http://doi.org/10.1037/bul0000054

Heimberg, R. G., Mueller, G. P., Holt, C. S., Hope, D. A., \& Liebowitz, M. R. (1992). Assessment of anxiety in social interaction and being observed by others: The social interaction anxiety scale and the Social Phobia Scale. Behavior Therapy, 23(1), 5373. http://doi.org/10.1016/S0005-7894(05)80308-9

Hesselmann, G., \& Moors, P. (2015). Definitely maybe: can unconscious processes perform the same functions as conscious processes? Consciousness Research, 584. http://doi.org/10.3389/fpsyg.2015.00584 
EMOTION AND ATTENTION

842 Jiang, Y., Costello, P., \& He, S. (2007). Processing of invisible stimuli: Advantage of upright

843 faces and recognizable words in overcoming interocular suppression. Psychological

844 science, $18(4), 349-355$.

845 Jiang, Y., \& He, S. (2006). Cortical responses to invisible faces: dissociating subsystems for

846 facial-information processing. Current Biology: CB, 16(20), 2023-2029.

$847 \quad$ http://doi.org/10.1016/j.cub.2006.08.084

848 Kim, C.-Y., \& Blake, R. (2005). Psychophysical magic: rendering the visible 'invisible'.

849 Trends in Cognitive Sciences, 9(8), 381-388. http://doi.org/10.1016/j.tics.2005.06.012

850 Koch, C. (2004). The quest for consciousness: A neurobiological approach (1st ed.). Denver,

851 CO: Roberts \& Company Publishers.

852 Koster, E. H. W., Verschuere, B., Burssens, B., Custers, R., \& Crombez, G. (2007). Attention

853

854

855

856

857

858

859

860

861

862

863

864

865

866

867 for emotional faces under restricted awareness revisited: do emotional faces automatically attract attention? Emotion (Washington, D.C.), 7(2), 285-295. http://doi.org/10.1037/1528-3542.7.2.285

Lange, C., 1885. The mechanism of the emotions. In: Dunlap, E. (Ed.), The Emotions. Williams \& Wilkins, Baltimore, Maryland, pp. 33-92.

Lapate, R. C., Rokers, B., Li, T., \& Davidson, R. J. (2013). Nonconscious Emotional Activation Colors First Impressions A Regulatory Role for Conscious Awareness. Psychological Science, 956797613503175. http://doi.org/10.1177/0956797613503175

LeDoux, J. (1998). The emotional brain: The mysterious underpinnings of emotional life. Simon and Schuster.

Lee, D. H., Susskind, J. M., \& Anderson, A. K. (2013). Social transmission of the sensory benefits of eye widening in fear expressions. Psychological Science, 24(6), 957-965.

Mogg, K., \& Bradley, B. P. (1998). A cognitive-motivational analysis of anxiety. Behaviour Research and Therapy, 36(9), 809-848. http://doi.org/10.1016/S00057967(98)00063-1 
EMOTION AND ATTENTION

868 Mogg, K., \& Bradley, B. P. (1999). Orienting of Attention to Threatening Facial Expressions

869

870

871

872

873

874

875

876

877

878

879

880

881

882

883

884

885

886

887

888

889

890

891

892 Presented under Conditions of Restricted Awareness. Cognition and Emotion, 13(6), 713-740. http://doi.org/10.1080/026999399379050

Mogg, K., \& Bradley, B. P. (2002). Selective orienting of attention to masked threat faces in social anxiety. Behaviour Research and Therapy, 40(12), 1403-1414.

Mogg, K., Bradley, B. P., \& Hallowell, N. (1994). Attentional bias to threat: roles of trait anxiety, stressful events, and awareness. The Quarterly Journal of Experimental Psychology. A, Human Experimental Psychology, 47(4), 841-864.

Morris, J. S., Öhman, A., \& Dolan, R. J. (1999). A subcortical pathway to the right amygdala mediating 'unseen' fear. Proceedings of the National Academy of Sciences of the United States of America, 96(4), 1680-1685.

Murphy, S. E., Downham, C., Cowen, P. J., \& Harmer, C. J. (2008). Direct effects of diazepam on emotional processing in healthy volunteers. Psychopharmacology, 199(4), 503-513. http://doi.org/10.1007/s00213-008-1082-2

Ohman, A., \& Mineka, S. (2001). Fears, phobias, and preparedness: toward an evolved module of fear and fear learning. Psychological Review, 108(3), 483-522.

Öhman, A., Flykt, A., \& Esteves, F. (2001). Emotion drives attention: detecting the snake in the grass. Journal of Experimental Psychology: General, 130(3), 466-478.

Ohman, A., \& Soares, J. J. (1994). 'Unconscious anxiety': phobic responses to masked stimuli. Journal of Abnormal Psychology, 103(2), 231-240.

Pessoa, L., Japee, S., Sturman, D., \& Ungerleider, L. G. (2006). Target visibility and visual awareness modulate amygdala responses to fearful faces. Cerebral Cortex (New York, N.Y.: 1991), 16(3), 366-375. http://doi.org/10.1093/cercor/bhi115

Sekar, K., Findley, W. M., Poeppel, D., \& Llinás, R. R. (2013). Cortical response tracking the conscious experience of threshold duration visual stimuli indicates visual perception 
EMOTION AND ATTENTION

893

894

895

896

897

898

899

900

901

902

903

904

905

906

907

908

909

910

911

912

913

914

915

is all or none. Proceedings of the National Academy of Sciences, 110(14), 5642-5647. http://doi.org/10.1073/pnas.1302229110

Shanks, D. R. (2017). Regressive research: The pitfalls of post hoc data selection in the study of unconscious mental processes. Psychonomic Bulletin \& Review, 24(3), 752-775.

Sklar, A. Y., Levy, N., Goldstein, A., Mandel, R., Maril, A., \& Hassin, R. R. (2012). Reading and doing arithmetic nonconsciously. Proceedings of the National Academy of Sciences, 109(48), 19614-19619. http://doi.org/10.1073/pnas.1211645109

Skrandies W. (1987). “The upper and lower visual field of man: Electrophysiological and functional differences". In: Ottoson D. (Ed.), Progress in Sensory Physiology. Berlin: Springer;

Stein, T., \& Sterzer, P. (2012). Not just another face in the crowd: detecting emotional schematic faces during continuous flash suppression. Emotion (Washington, D.C.), 12(5), 988-996. http://doi.org/10.1037/a0026944

Spielberger, C. D., Gorsuch, R. L., Lushene, R., Vagg, P. R., \& Jacobs, G. A. (1983). Manual for the State-Trait Anxiety Inventory. Palo Alto, CA: Consulting Psychologists Press.

Szczepanowski, R., \& Pessoa, L. (2007). Fear perception: Can objective and subjective awareness measures be dissociated? Journal of Vision, 7(4), 10-10. http://doi.org/10.1167/7.4.10

Tamietto, M., \& de Gelder, B. (2010). Neural bases of the non-conscious perception of emotional signals. Nature Reviews Neuroscience, 11(10), 697-709. http://doi.org/10.1038/nrn2889

Tsuchiya, N., \& Koch, C. (2005). Continuous flash suppression reduces negative afterimages. Nature Neuroscience, 8(8), 1096-1101. http://doi.org/10.1038/nn1500 
EMOTION AND ATTENTION

916 Tsuchiya, N., Moradi, F., Felsen, C., Yamazaki, M., \& Adolphs, R. (2009). Intact rapid

917

918

919

920

921

922

923

924

925

926

927

928

929

930

931

932

933

934

935

936

detection of fearful faces in the absence of the amygdala. Nature Neuroscience, 12(10), 1224-1225. http://doi.org/10.1038/nn.2380

Vuilleumier, P., \& Schwartz, S. (2001). Emotional facial expressions capture attention. Neurology, 56(2), 153-158.

Whalen, P. J., Kagan, J., Cook, R. G., Davis, F. C., Kim, H., Polis, S., ... Johnstone, T. (2004). Human Amygdala Responsivity to Masked Fearful Eye Whites. Science, 306(5704), 2061-2061. http://doi.org/10.1126/science.1103617

Wiens, S. (2008). Concepts of visual consciousness and their measurement. Advances in Cognitive Psychology, 3(1-2), 349-359. http://doi.org/10.2478/v10053-008-0035-y

Wiens, S., \& Öhman, A. (2007). Probing unconscious emotional processes: On becoming a successful masketeer. In J. A. Coan \& J. J. B. Allen (Eds.), Handbook of emotion elicitation and assessment (pp. 65-90). New York, NY: Oxford University Press.

Williams, M. A., Morris, A. P., McGlone, F., Abbott, D. F., \& Mattingley, J. B. (2004). Amygdala responses to fearful and happy facial expressions under conditions of binocular suppression. The Journal of Neuroscience: The Official Journal of the Society for Neuroscience, 24(12), 2898-2904.

http://doi.org/10.1523/JNEUROSCI.4977-03.2004 
EMOTION AND ATTENTION

937

Footnotes

$938{ }^{1}$ Notably, not all authors have claimed that observers were completely unaware of the

939 masked stimuli, and have instead claimed that awareness has been "restricted" (e.g. Carlson

940 \& Reinke, 2008; Mogg \& Bradley, 1999). Nonetheless, it remains a matter of contention,

941 with theoretical importance, to determine whether emotionally salient stimuli attract attention

942 under genuine conditions of unawareness.

943 